\title{
Monte Carlo simulation of light transport in dark-field confocal photoacoustic microscopy
}

Zhixing Xie, Lihong V. Wang, Hao F. Zhang

Zhixing Xie, Lihong V. Wang, Hao F. Zhang, "Monte Carlo simulation of light transport in dark-field confocal photoacoustic microscopy," Proc. SPIE 7177, Photons Plus Ultrasound: Imaging and Sensing 2009, 717717 (24 February 2009); doi: 10.1117/12.810254

SPIE. Event: SPIE BiOS, 2009, San Jose, California, United States 


\title{
Monte Carlo simulation of light transport in dark-field confocal photoacoustic microscopy
}

\author{
Zhixing Xie ${ }^{\mathrm{a}}$, Lihong V. Wang ${ }^{\mathrm{b}}$, Hao F. Zhang $\square^{\mathrm{a}}$ \\ ${ }^{a}$ Functional Optical Imaging Lab, Department of Electrical Engineering \& Computer Science, The \\ University of Wisconsin-Milwaukee, Milwaukee, WI 53201; \\ ${ }^{b}$ Optical Imaging Laboratory, Department of Biomedical Engineering, Washington University \\ in St. Louis, St. Louis, MO 63130
}

\begin{abstract}
A modified MC convolution method for integration extension of MC simulation is developed for finite photon beam with random shape of translational or rotational invariance, which is proven consistent with the conventional convolution extension of MC simulation for normal incident finite beam. The method is applied to analyze the positions of fluence foci and ratios of fluence at the focus and surface which are two key factors in the application of dark-field confocal and some interesting points are presented including: 1) The fluence profile has a saddle-like shape with highest peak in the bright field and low valley near the surface and a second rise in the center of dark field which is defined as the effective optical focus; 2) Besides a little peak near zero inner radius, the ratio of fluences at the focus and surface increases linearly with the inner radius, suggesting the large inner radius more advantageous to image at the effective optical focus; 3) The position of effective optical foci deepens linearly with the increase of the inner radius, suggesting that to get a high quality image of deeper target, a dark-field with larger size is more beneficial. But the position of fluence foci are far away from the foci of geometrical laser beam in high scattering tissue, so aligning the foci of geometrical laser beam and acoustic transducer doesn't guarantee that effective optical focus is accurately overlapping with the acoustic focus. An MC simulation with integration extension presented in this paper maybe helpful to determine where the acoustic focus should be to maximize the SNR in tissue imaging; 4) incident angle makes little difference to ratio of fluences at the focus and surface and an incident angle between 30 and 50 degrees gives the highest fluence at the effective optical focus; 5) the depth of fluence focus is insensitive to the incident angle.
\end{abstract}

Keywords: Monte Carlo simulation, photoacoustic microscopy, dark-field illumination

\section{INTRODUCTION}

In the past decade, photoacoustic (PA) imaging [1,2] has been investigated intensively and been applied to in vivo imaging of tissue anatomy, tumors, burns, hemodynamic changes, and gene expression. Among all the existing PA imaging technologies, functional photoacoustic microscopy (PAM) has shown great potential in imaging subcutaneous structure and functions [3-7]. It employs a dark-field illumination, where the excitation laser beam has a ring-shaped cross section, to avoid the reverberations due to overly strong PA signal generated from the skin surface. In addition, the optical-ultrasonic confocal geometry enables the weaker signal generated from deep structures can be detected with a high signal-to-noise ratio (SNR) by a high-sensitivity focused ultrasonic detector. Together with confocal microscopy, two-photon microscopy, and optical coherence tomography, PAM is considered as a high-resolution imaging modality as all of them can achieve a depth-to-resolution ratio greater than 100; however, PAM offers optical absorption contrast that is not available from other modalities.

In developing and optimizing PAM, two factors are critical to achieve a good SNR and avoid reverberation for highquality imaging. One is to keep the focal region of the acoustic transducer and weakly focused laser beam overlapping. In an optically turbid medium such as biological tissue, it is hard to implement because optical focusing is very inefficient beyond one transport mean free path in a turbid medium [8]. Another is to maximize the ratio of the fluence between the effective optical focal region and surface along the axis of the ultrasonic detection. An accurate model of

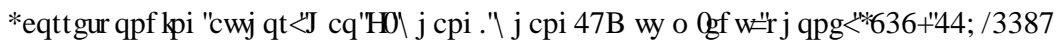

Photons Plus Ultrasound: Imaging and Sensing 2009, edited by Alexander A. Oraevsky, Lihong V. Wang, Proc. of SPIE Vol. 7177, 717717 - (C) 2009 SPIE · CCC code: 1605-7422/09/\$18 - doi: 10.1117/12.810254 
light transport of dark-field confocal PAM is essential to understanding these two factors. The Monte Carlo (MC) method [9-12] provides a flexible tool to model light transport in turbid media [13-16].

Previous Monte Carlo simulations with the focused beam configurations have mainly provided the understanding of the effects of scattering and absorption parameters and surface roughness on the fluence distribution with incident beam in the shape of solid cone $[8,17,18]$, although in these studies by tracking all of photon packages incident on the interface, the methods employed can be extended to the incident beam of arbitrary shape in principle. However in order to keep the statistical error from growing, the number of photon packages being tracked and computation burden have to explode as square as the size of illumination pattern. There are some existing accelerating methods designed to break the limits of the feature of square-increasing computation such as convolution [19] suitable to beam with illumination shape of translational invariance, scaling method [20] suitable to beam with invariant illumination shape and different sets of optical properties of media. But so far because of lack of an accelerating method suitable to beam with both translational and rotational invariance, to our knowledge, the systemic understanding of the effect of illumination pattern with large variation range of size and incident angle on the fluence distribution has remained a challenge. As to the case of darkfield confocal PAM, in process of imaging, as long as the sample is determined, the scattering and absorption optical parameters and surface roughness are fixed, how to position and adjust the conical lens to choose illumination pattern, specifically the form of obliquely incident annular beam to get the best imaging quality has been wrestling in the mind of experimenters. So in this paper, different with the previous studies, we developed a modified MC convolution method for integration extension of MC simulation breaking the limits of the feature of square-increasing computation and suitable to finite illumination beams with rotational and translational invariance, and applied the method focusing on the influences of the illumination patterns on fluence distribution and the two key factors in the case of dark-field confocal PAM.

\section{METHODS}

\subsection{Invariance relation and integration method}

Let's assume that the response of an oblique-incident pencil beam (Green's function) at the origin of a coordinate system is $G_{p}(r, \theta, z)$ in a cylindrical coordinate system and $G_{d}(x, y, z)$ in a Cartesian coordinate system. The Green's function is acquired from codes modified from the standard Monte Carlo simulation as detailed in [9] which can be obtained by one baseline run of MCML simulation. The impulse pencil beam is incident in the $x-z$ plane and the surface medium is in the $x-y$ plane. For the same impulse input, there is transformational invariance relation between two expressions of Green's functions as follows

$$
\begin{aligned}
& G_{d}(x, y, z)=G_{p}\left(\sqrt{x^{2}+y^{2}}, \arctan 2(x, y), z\right) \\
& G_{p}(r, \theta, z)=G_{d}(r \cos \theta, r \sin \theta, z)
\end{aligned}
$$

$$
\begin{aligned}
& \quad r=\sqrt{x^{2}+y^{2}}, \quad \cos \theta=x / \sqrt{x^{2}+y^{2}}, \quad \sin \theta=y / \sqrt{x^{2}+y^{2}} \quad \text { and } \\
& \arctan 2(x, y)=\arctan \frac{y}{x}+(1-\operatorname{sgn}(x)) \operatorname{sgn}(y) \frac{\pi}{2} \text {. Here, } \arctan 2(x, y) \quad \text { denotes the arctangent of } \frac{y}{x} \text { taking }
\end{aligned}
$$
into account which quadrant the point $(x, y)$ is in and $\operatorname{sgn}()$ is the sign function.

For the pencil beam rotating an angle $\theta^{\prime}$ along the $\mathrm{z}$ axis as shown in Figure 1a, the green functions for the responses obey the rotational invariance relation written as

$$
G_{p}\left(r, \theta, z: \theta^{\prime}\right)=G_{p}\left(r, \theta-\theta^{\prime}, z\right)=G_{d}\left(r \cos \left(\theta-\theta^{\prime}\right), r \sin \left(\theta-\theta^{\prime}\right), z\right)=G_{d}\left(x^{\prime}, y^{\prime}, z\right),
$$

where $x^{\prime}=r \cos \left(\theta-\theta^{\prime}\right)$ and $y^{\prime}=r \sin \left(\theta-\theta^{\prime}\right)$. 


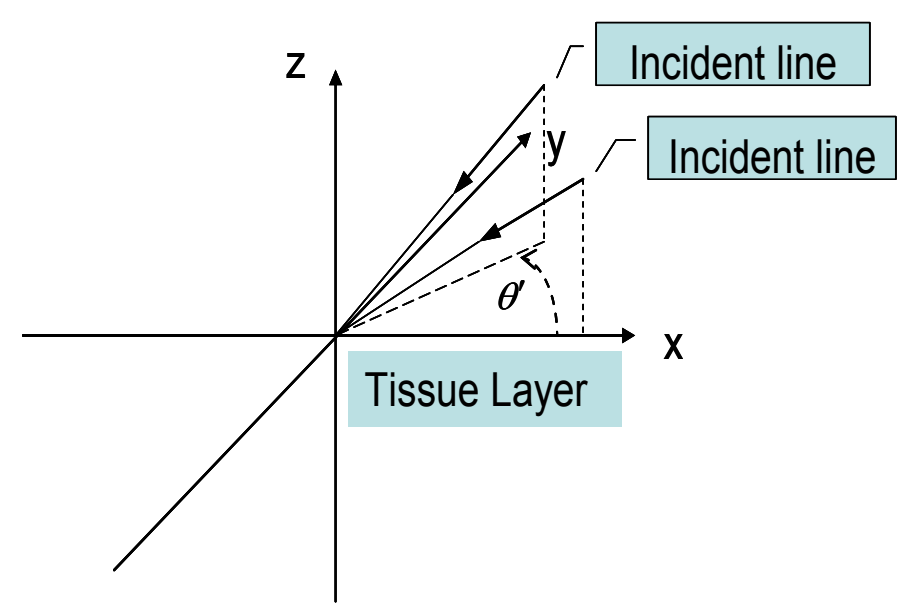

(a) rotation

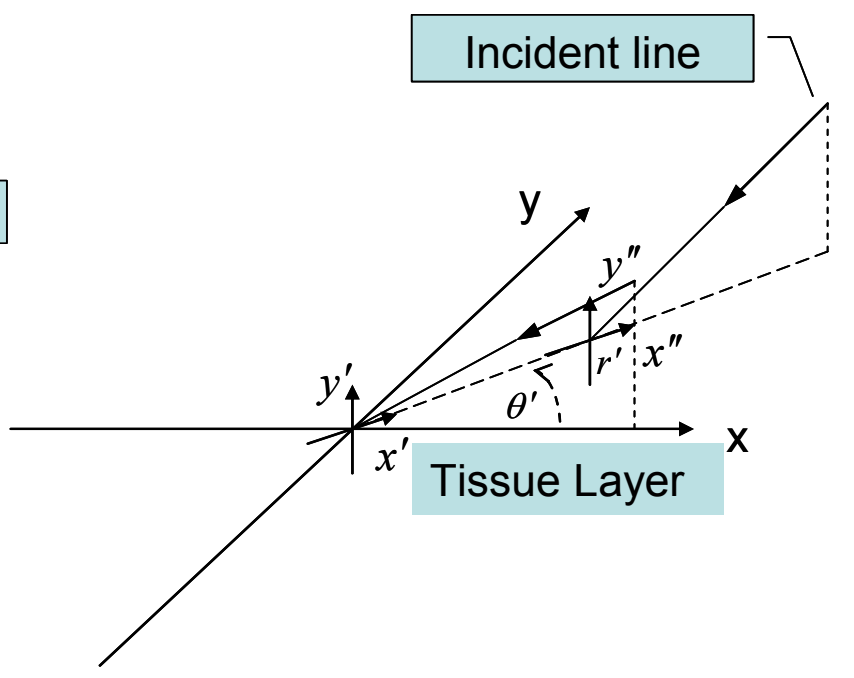

(b) rotation and translation

Figure 1. The schematic of rotation and translation of the impulse pencil beam.

For the pencil beam translating in the xy plane with no change of incident angle, the green functions for the responses keep the translational invariance relation which is just convolution relation of MC simulation. In the situation shown in Figure $1 \mathrm{~b}$, after rotating an angle $\theta^{\prime}$, the pencil beam further translates a distance $r^{\prime}$ along $x^{\prime}$ axis, the translational invariance here can be described as

$$
G_{d}\left(x, y, z: r^{\prime}, \theta^{\prime}\right)=G_{d}\left(x^{\prime}, y^{\prime}, z: r^{\prime}\right)=G_{d}\left(x^{\prime}-r^{\prime}, y^{\prime}, z\right)=G_{d}\left(r \cos \left(\theta-\theta^{\prime}\right)-r^{\prime}, r \sin \left(\theta-\theta^{\prime}\right), z\right)(3)
$$

Combining Eq. 3 with Eq. 1, the invariance relation after rotating and translating from origin to site $\left(r^{\prime}, \theta^{\prime}\right)$ can be expressed in the cylindrical coordinate as

$$
G_{p}\left(r, \theta, z: r^{\prime}, \theta^{\prime}\right)=G_{p}\left(\sqrt{r^{2}+r^{\prime 2}-2 r r^{\prime} \cos \left(\theta-\theta^{\prime}\right)}, \arctan 2\left(r \cos \left(\theta-\theta^{\prime}\right)-r^{\prime}, r \sin \left(\theta-\theta^{\prime}\right)\right), z\right)(4)
$$

Here $G_{p}\left(r, \theta, z: r^{\prime}, \theta^{\prime}\right)$ is the Green's function for the response of the incident pencil beam at the site $\left(r^{\prime}, \theta^{\prime}\right)$ which keeps the rotational and translational invariance relations, namely, the angle between the laser beam and normal to the incident surface being constant throughout the movement.

Assuming the finite photon beam as the source has the intensity profile $s(x, y)$ or $s(r, \theta)$ at the area $\mathrm{S}$ of the incident interface with the random shape keeping translational invariance relation and rotational invariance relation. The response $C_{p}(r, \theta, z)$ can be obtained through integrating the distribution function $G_{p}\left(r, \theta, z: r^{\prime}, \theta^{\prime}\right)$ around the illumination area $S$ as follows,

$$
C_{p}(r, \theta, z)=\iint_{S} G_{p}\left(r, \theta, z: r^{\prime}, \theta^{\prime}\right) s\left(r^{\prime}, \theta^{\prime}\right) d S
$$

\subsection{Beam with the shape of only parallel translation}

In this case, there is no change of incident direction at the illumination interface as shown in Figure 2a. The translational invariance relation can be written as

$$
G_{d}\left(x, y, z: x^{\prime}, y^{\prime}\right)=G_{d}\left(x-x^{\prime}, y-y^{\prime}, z\right)
$$



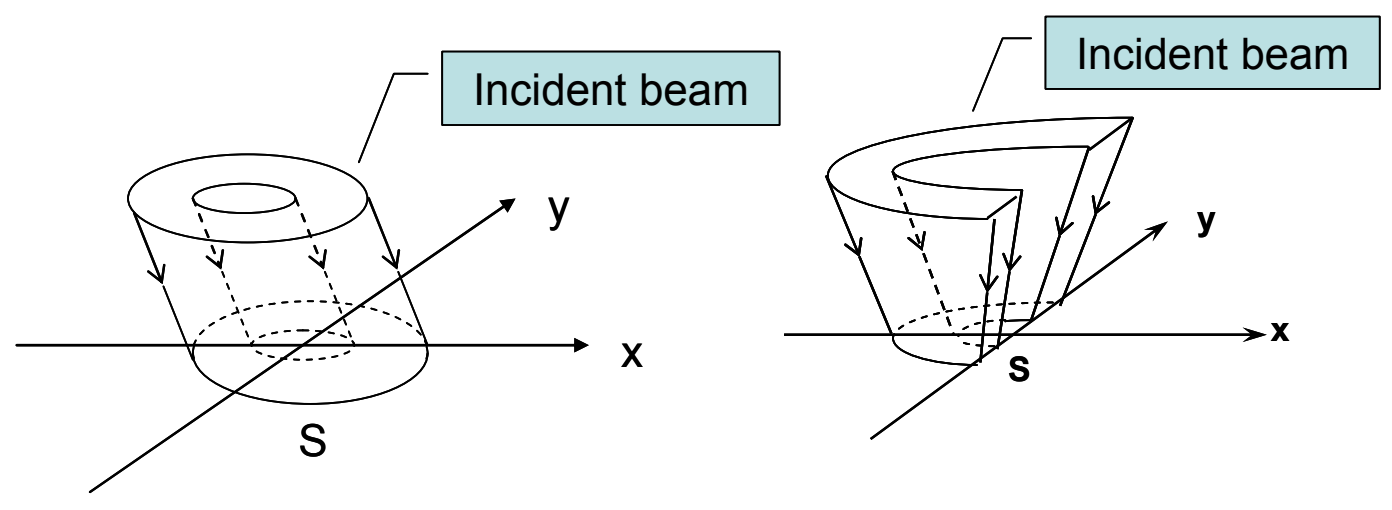

(a) Parallel translation

(b) Rotation and translation

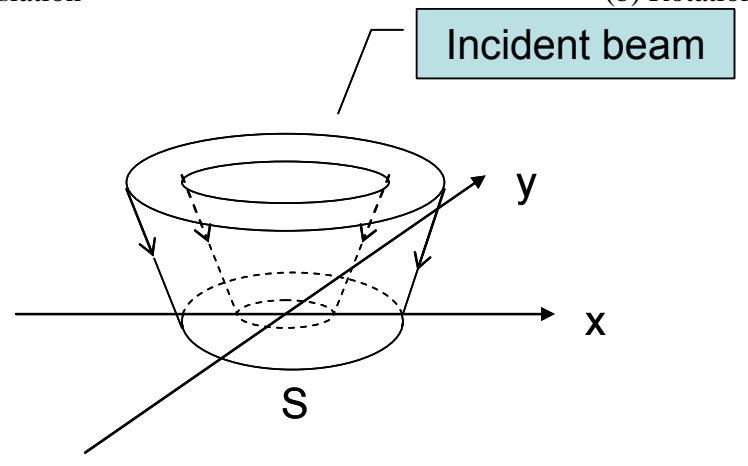

(c) Dark field confocal PAM

Figure 2. The schematic of the finite laser beams. (a) With the shape of parallel translation between pencil beams; (b) With the shape of rotation and translation between pencil beams; (C) With the cylindrically symmetric shape of rotation and translation between pencil beams in dark-field confocal PAM.

Combining Eq. 1 with Eq. 6, we have

$G_{p}\left(r, \theta, z: r^{\prime}, \theta^{\prime}\right)=G_{p}\left(\sqrt{r^{2}+r^{\prime 2}-2 r r^{\prime} \cos \left(\theta-\theta^{\prime}\right)}, \arctan 2\left(r \cos \theta-r^{\prime} \cos \theta^{\prime}, r \sin \theta-r^{\prime} \sin \theta^{\prime}\right), z\right)(7)$

The response for the beam with the shape of only translational invariance can be obtained through integrating the distribution function $G_{d}\left(x, y, z: x^{\prime}, y^{\prime}\right)$ or $G_{p}\left(r, \theta, z: r^{\prime}, \theta^{\prime}\right)$ around the illumination area $S$ as follows,

$$
C_{d}(x, y, z)=\iint_{S} G_{d}\left(x-x^{\prime}, y-y^{\prime}, z\right) s\left(x^{\prime}, y^{\prime}\right) d x^{\prime} d y^{\prime}
$$

$C_{p}(r, \theta, z)$

$=\iint_{S} G_{p}\left(\sqrt{r^{2}+r^{\prime 2}-2 r r^{\prime} \cos \left(\theta-\theta^{\prime}\right)}, \arctan 2\left(r \cos \theta-r^{\prime} \cos \theta^{\prime}, r \sin \theta-r^{\prime} \sin \theta^{\prime}\right), z\right) s\left(r^{\prime}, \theta^{\prime}\right) r^{\prime} d \theta^{\prime} d r^{\prime}$

If the beam is incident normally to the surface, $G_{p}$ is cylindrically symmetric. Thus, Eq. 8 can be simplified as

$$
C_{p}(r, \theta, z)=\iint_{S} G_{p}\left(\sqrt{r^{2}+r^{\prime 2}-2 r r^{\prime} \cos \left(\theta-\theta^{\prime}\right)}, z\right) s\left(r^{\prime}, \theta^{\prime}\right) r^{\prime} d \theta^{\prime} d r^{\prime},
$$

which is the same as the convolution formula given [19]. 


\subsection{Beam with the shape of both rotation along the normal of the interface and translation}

In this case, the incident direction changes at the different site of the interface but keeping a constant angle with the normal of the interface as shown in Figure 2b. Utilizing the rotational and translational invariance relations as presented in Eq. 4, combined with the Eqs. 1 and 5, the response for the beam with the shape of both rotation along the normal of the interface and translation can be expressed as

$$
\begin{gathered}
C_{d}(x, y, z)=\iint_{S} G_{d}\left(\frac{x x^{\prime}+y y^{\prime}-\left(x^{\prime 2}+y^{\prime 2}\right)}{\sqrt{x^{\prime 2}+y^{\prime 2}}}, \frac{y x^{\prime}-x y^{\prime}}{\sqrt{x^{\prime 2}+y^{\prime 2}}}, z\right) s\left(x^{\prime}, y^{\prime}\right) d x^{\prime} d y^{\prime} \\
C_{p}(r, \theta, z)=\iint_{S} G_{p}\left(\sqrt{r^{2}+r^{\prime 2}-2 r r^{\prime} \cos \left(\theta-\theta^{\prime}\right)}, \arctan 2\left(r \cos \left(\theta-\theta^{\prime}\right)-r^{\prime}, r \sin \left(\theta-\theta^{\prime}\right)\right), z\right) s\left(r^{\prime}, \theta^{\prime}\right) r^{\prime} d \theta^{\prime} d r^{\prime}
\end{gathered}
$$

\subsection{Beam in dark-field confocal PAM}

In the case of dark-field confocal PAM, the laser beam profile is cylindrically symmetrical while keeping the rotational and translational invariance as shown in Figure 2c, so only the response along the positive $\mathrm{x}$ axis needs to be integrated, namely,

$$
C_{p}(r, z)=C_{d}(r, 0, z)
$$

With the reference of Eq. 11 and Eq. 1, the response expression of Eq. 10 can be simplified as

$$
C_{p}(r, z)=\iint_{S} G_{d}\left(r \cos \theta^{\prime}-r^{\prime},-r \sin \theta^{\prime}, z\right) s\left(r^{\prime}, \theta^{\prime}\right) r^{\prime} d \theta^{\prime} d r^{\prime}
$$

Here it should be noted that $G_{d}(x, y, z)$ is the Green's function for the response of the pencil beam obtained by MC simulation and presented in Cartesian coordinate system, the intensity profile of source beam $s(r, \theta)$ and its response $C_{p}(r, z)$ are presented in cylindrical coordinate system, and the integral is carried out along cylindrical coordinates. So in the numerical solution to the integration, interpolation is used to evaluate the values of $G_{d}$ at the integrating grid points from the values at original Cartesian discrete grid points of MC simulation.

\section{SAMPLE SIMULATION}

In this section, we will present some sample runs of integration extension of MC simulation for the beam in dark-field confocal PAM. A two-layered skin model was assumed with optical properties listed in Table 1 . The refractive indices for the top and bottom ambient media are 1.33 on account of water usually used as the acoustic coupler between the acoustic transducer and target tissue in dark-field confocal PAM. The Green's function for the baseline pencil beam was acquired from codes modified from the well-known MCML program, as detailed in [9]. The grid size along all axes is $0.005 \mathrm{~cm}$. The number of grid elements in the $\mathrm{x}, \mathrm{y}$ and $\mathrm{z}$ directions are 400, 200 and 200 respectively. The baseline beam is incident at the origin and in the xz plane with its response symmetric with respect to xz plane, so 200 grid elements along the y axis only cover the range from $-0.01 \mathrm{~mm}$ to $0.99 \mathrm{~cm}$. The number of photon packets traced is 30 million. In numerical solution for Eqs 8 and 12, a 2D linear algorithm for interpolation and an adaptive Simpson algorithm for quadrature are assumed with the integration tolerance of 0.0001 .

Table 1. The optical properties of the two-layer tissue model in the MC simulation. The refractive indices for the top and bottom ambient media are 1.33 .

\begin{tabular}{|c|c|c|c|c|c|}
\hline Layer & $\mathrm{n}$ & $\mu_{a}\left[\mathrm{~cm}^{-1}\right]$ & $\mu_{s}\left(\mathrm{~cm}^{-1}\right)$ & $\mathrm{g}[-]$ & Thickness $(\mathrm{cm})$ \\
\hline Epidermis & 1.34 & 0.15 & 400 & 0.85 & 0.001 \\
\hline Dermis & 1.4 & 0.7 & 200 & 0.76 & 2 \\
\hline
\end{tabular}


In the first place, a normal incident circular flat beam with an energy intensity $1 \mathrm{~mJ} / \mathrm{cm}^{2}$ at the illuminated surface and a radius of $0.2 \mathrm{~cm}$ was adopted to check the internal consistency between the conventional convolution and integration extension to MC simulation. Because of the cylindrical symmetry of the fluence distribution of baseline pencil beam in this case, the conventional convolution with Eqs. 8 and integration extension with Eq. 12 are both suitable to calculate the total response of the finite beam. The result of conventional convolution acquired from the published CONV program [19] and that of integration extension in our calculations have been proven identical and shown in Figure 3a. The fluence profile has a top-hat shape with only high peak in the center of bright field near the surface and then diffuses and deceases rapidly out of the illuminated area along $r$ and $\mathrm{z}$ directions.

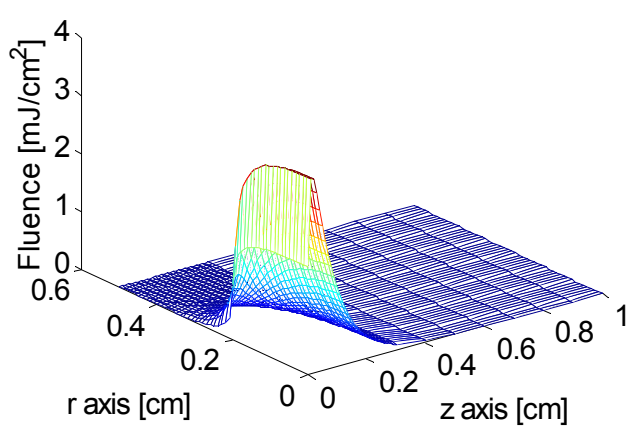

(a)

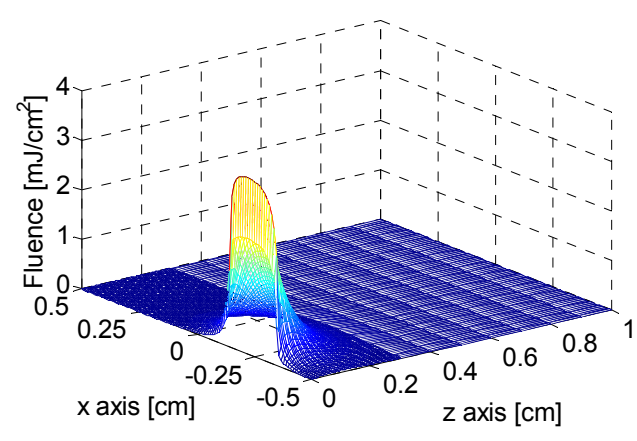

(c)

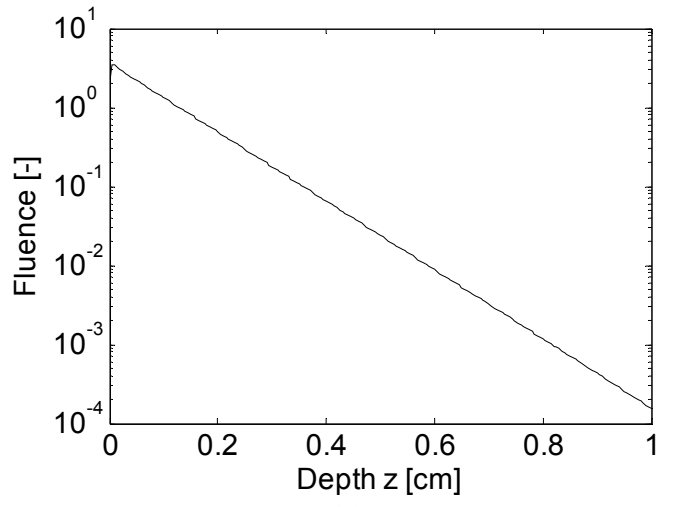

(e)

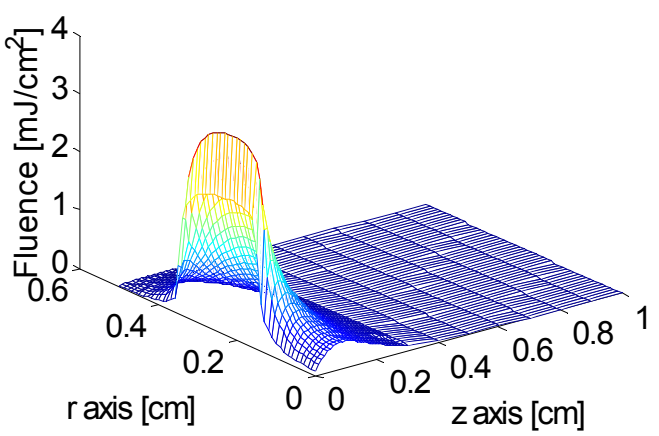

(b)

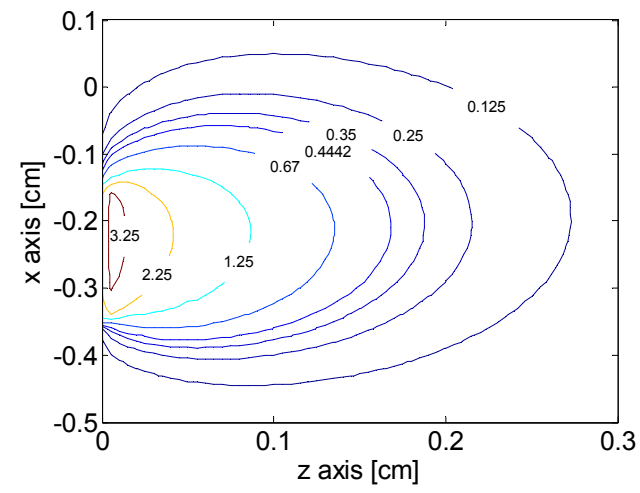

(d)

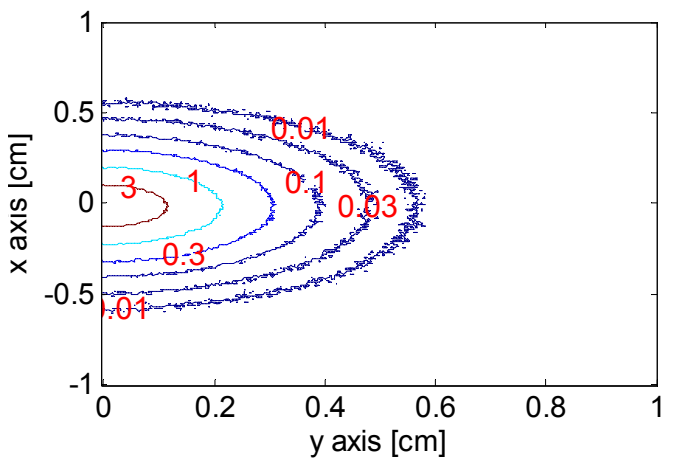

(f) 
Figure 3. (a)2-Dimensional fluence distribution along the $\mathrm{r}$ and $\mathrm{z}$ directions for the circular flat beam with normal incident angle and $2 \mathrm{~mm}$ radius for bright-field. (b) 2-Dimensional fluence distribution along the $\mathrm{r}$ and $\mathrm{z}$ directions for the obliquely and cylindrical-symmetrically finite beam with 45 degree incident angle and $1.5 \mathrm{~mm}$ inner radius for darkfield and $3.5 \mathrm{~mm}$ outer radius for bright-field (c) 2-Dimensional fluence distribution along the $\mathrm{x}$ and $\mathrm{z}$ directions for the obliquely half angular finite beam with 45 degree incident angle and $1.5 \mathrm{~mm}$ inner radius for dark-field and $3.5 \mathrm{~mm}$ outer radius for bright-field (d) Contour view of fluence distribution in the x-z plane for the obliquely half angular finite beam with 45 degree incident angle and $1.5 \mathrm{~mm}$ inner radius for dark-field and $3.5 \mathrm{~mm}$ outer radius for brightfield (e) Dimensionless Internal fluence summing in the $\mathrm{x}-\mathrm{y}$ plane versus the $\mathrm{z}$ axis of the oblique impulse pencil beam with 45 degree angle (f) Contour view of fluence distribution in the $x-y$ plane at the depth $z=0.2 \mathrm{~cm}$ for the impulse pencil beam.

The response of fluence for the obliquely and cylindrical-symmetrically finite beam typical in dark-field confocal PAM with 45 degree incident angle, 1.5mm inner radius for dark-field, $3.5 \mathrm{~mm}$ outer radius for bright-field and an energy intensity $1 \mathrm{~mJ} / \mathrm{cm}^{2}$ at the illuminated surface was calculated using integration extension with Eq. 12 and shown in Figure 3b. The interesting point is that different from top-hat shape in the case of circular flat beam, the fluence profile in this case has a saddle-like shape with highest peak in the bright field and low valley near the surface and a second rise in the center of dark field. We define the position of the maximum in the second rise as the effective optical focus of the laser beam in the media, and analyze the relations of the positions of effective optical foci and geometrical optical foci of the incident laser beams and ratios of fluence at the focus and surface of the media in various conditions.

Figure 3c and 3d show the fluence distribution and its contour view along the $x-z$ plane for the obliquely half angular finite beam with 45 degree incident angle, $1.5 \mathrm{~mm}$ inner radius for dark-field, $3.5 \mathrm{~mm}$ outer radius for bright-field and an energy intensity $1 \mathrm{~mJ} / \mathrm{cm}^{2}$ at the illuminated surface. We can see that the peak of fluence appears far from the origin center of illumination area, so it belongs to a kind of dark-field illumination. With the increase of the depth, the fluence diffuses to the central region. Video 1 gives the contour view of the fluence distribution along the $x-y$ plane and its propagation with the increase of the depth. We can see that the shape near the surface is half angular, just similar to the illumination pattern, with the increase of the depth the shape of fluence distribution turns into ellipse.

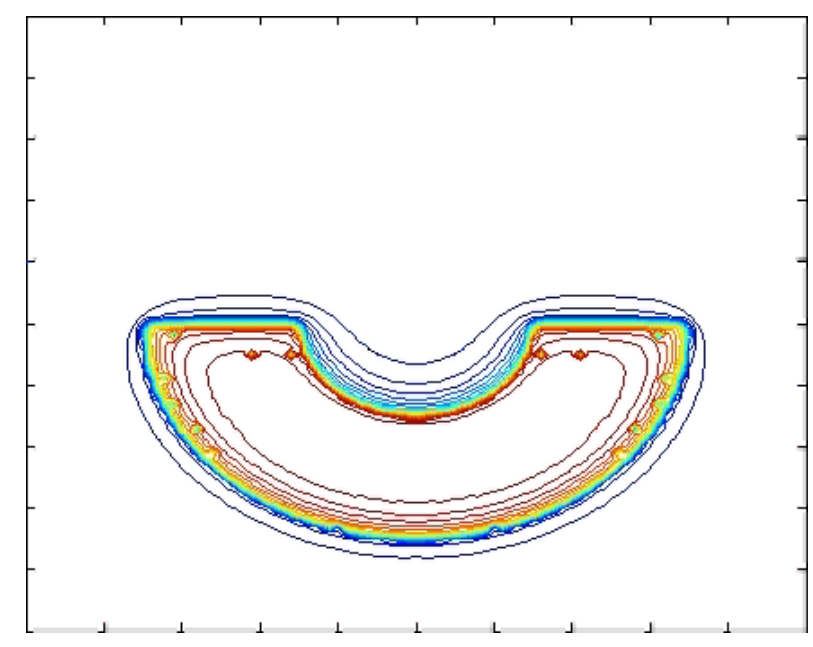

Video 4. The movie shows the contour view of fluence distribution along the $x-y$ plane and its propagation with the increase of the depth for the obliquely half angular finite beam with 45 degree incident angle and $1.5 \mathrm{~mm}$ inner radius for dark-

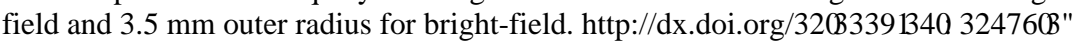

Figure 3e indicates the dimensionless internal fluence as a function of depth $\mathrm{z}$ for the oblique baseline pencil beam with 45 degree incident angle which is just similar with that of the normal incident impulse beam in the previous simulations [9]. But from the contour view of fluence distribution of the pencil beam at the depth $z=0.2 \mathrm{~cm}$ shown in Figure $3 f$, the location of the peak fluence shifted from the origin along the $x$ axis by a distance less than $0.04 \mathrm{~cm}$. In comparison, the geometric shift should be $0.2 \mathrm{~cm}$ at the same depth in an optically clear medium. These facts suggest that in PAM the positions of the effective optical focus and the geometrical optical focus will differ from each other conspicuously in deep tissue. 


\section{DISCUSSION}

In the case of dark-field confocal PAM, the laser beam from the fiber passes through a conical lens to form a ring-shaped illumination pattern which is weakly focused into the tissue with the focal region coaxially overlapping the transducer focus inside the tissue. In the measurement, the relative position between the transducer and conical lens is fixed, but the distance between them and tissue can be honed to make the interested area at specific depth be imaged with highest SNR. This situation has been simulated with the obliquely and cylindrical-symmetrically incident finite beam with a moving inner radius of $0 \sim 3 \mathrm{~mm}$ for dark field and constant beam width of $1.5 \mathrm{~mm}$ for bright field and incident angle of 45 degree. Figure 4a presents the fluences at the focus and surface as a function of inner radius. Instead of keeping a constant total energy of finite beam, a constant energy intensity $1 \mathrm{~mJ} / \mathrm{cm}^{2}$ at the illuminated surface is assumed here. Taking into account the total energy is limited by the safety regulations and American National Standard Institute safety standard for the fluence is $20 \mathrm{~mJ} / \mathrm{cm}^{2}$ in the visible spectrum [21], the assumption is reasonable and makes no difference to the results on ratio of fluences at the focus and surface as a function of inner radius shown in Figure $4 \mathrm{~b}$ and the position of fluence foci as a function of inner radius shown in Figure 4c. Besides a little peak around $0.02 \mathrm{~cm}$ inner radiuses, the ratio of fluences at the focus and surface increases linearly with the inner radius, suggesting the large inner radius more advantageous to image at the fluence focus. The position of fluence foci deepens linearly with the increase of the inner radius. It is important to note that the center of the geometrical optical focus ranges from 0.075 to $0.375 \mathrm{~cm}$ with the increase of inner radius, almost twice as deep as the effective optical focus in the conditions herein. So aligning geometrical optical focus with the ultrasonic focus in an optically clear medium doesn't warrant that the effective optical focus will accurately overlap with the acoustic focus in tissue. Our simulation may be helpful to determine where the ultrasonic focus should be placed to maximize SNR in tissue imaging.

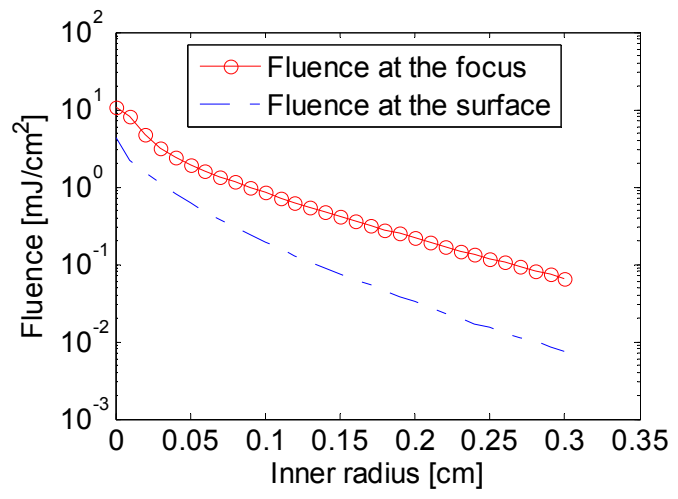

(a)

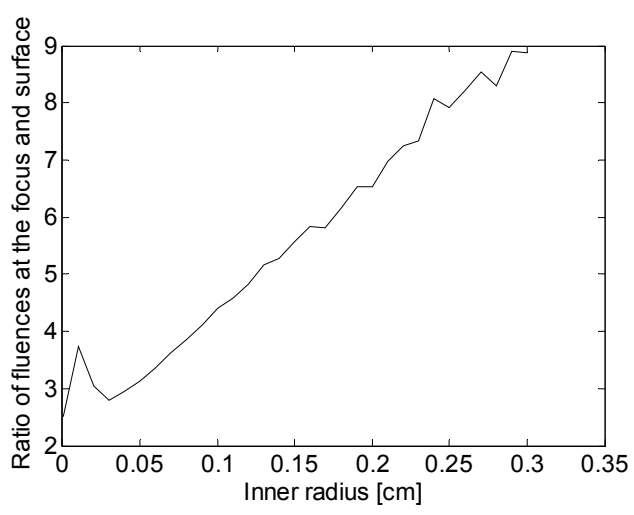

(b)

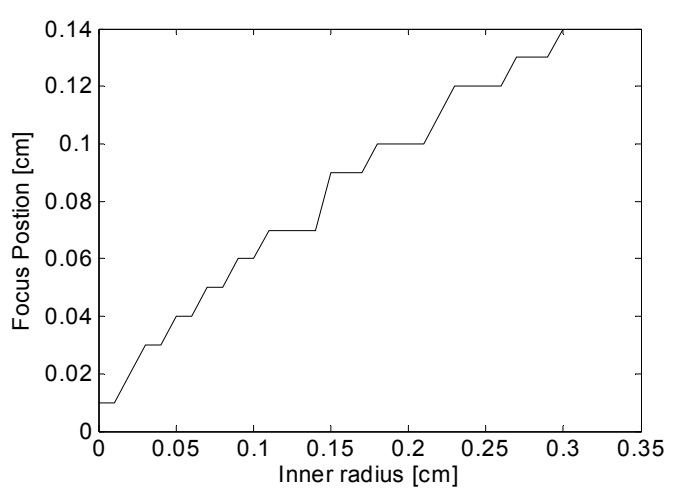

(c)

Figure 5. (a) Fluences at the effective optical focus and surface for the obliquely and cylindrical-symmetrically incident finite beam with a moving inner radius of $0 \sim 3 \mathrm{~mm}$ for dark-field and constant beam width of $1.5 \mathrm{~mm}$ for bright-field and incident angle of 45 degree. (b) Ratio of fluences at the focus and surface as a function of inner radius. (c) Position of effective optical foci as a function of inner radius. 
Another issue in the application of dark-field confocal PAM is which orientation of conical lens should be designed to get the best image. This situation has been simulated with the obliquely and cylindrical-symmetrically incident finite beam with $1 \mathrm{~mm}$ inner radius for dark-field and $2.5 \mathrm{~mm}$ outer radius for bright-field and incident angle ranging from 0 to 89 degree. Figure $5 a$ presents the fluences at the effective optical focus and surface as a function of incident angle. Again a constant energy intensity $1 \mathrm{~mJ} / \mathrm{cm}^{2}$ at the illuminated surface is assumed and the assumption doesn't affect the results on ratio of fluences at the focus and surface as a function of incident angle shown in Figure 5b and the position of effective optical foci as a function of incident angle shown in Figure 5c. Although a maximum fluence is reached at the effective optical focus at an incident angle of 35 degrees, angles between 30-50 degrees show almost the same fluence level $( \pm 3 \%)$. At an incident angle of $\sim 5$ degree, nearly normal incident angle provides best ratio of fluences at the focus and surface. The ratio decreases no more than $20 \%$ with the increase of the incident angle, and the position of fluence foci changes little from 0.07 to $0.05 \mathrm{~cm}$ in depth. Interestingly, the simulation indicates that incident angle makes little difference to image in dark-field confocal PAM and a smaller incident angle may be a little more advantageous to imaging quality, the depth of fluence focus is almost stable which can be obtained by MC simulation with integration extension in advance and apparently away from the geometrical optical focus.

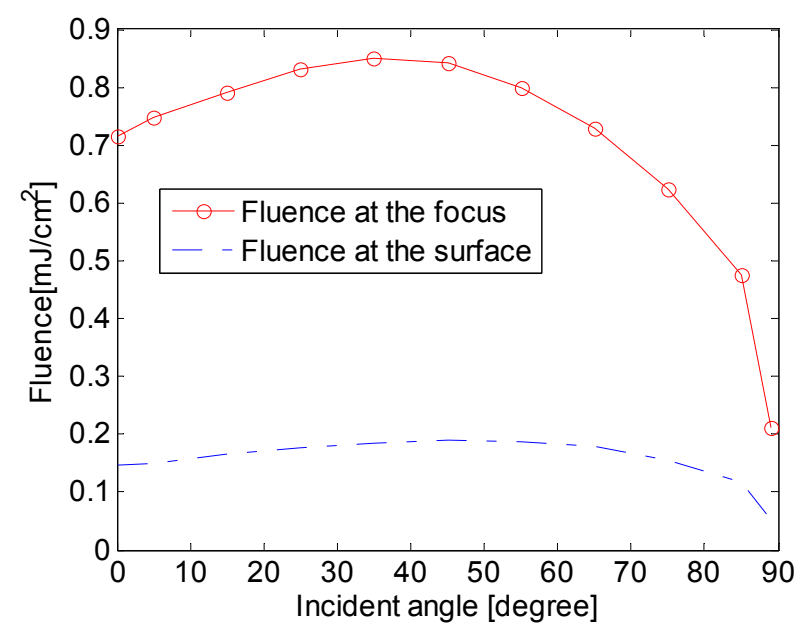

(a)

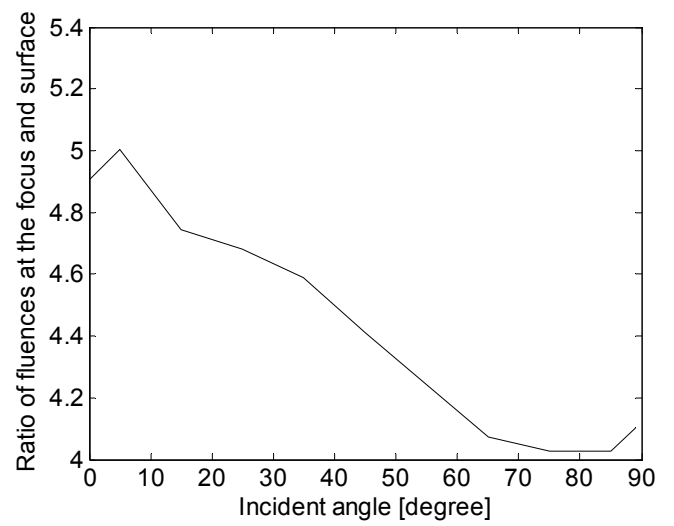

(b)

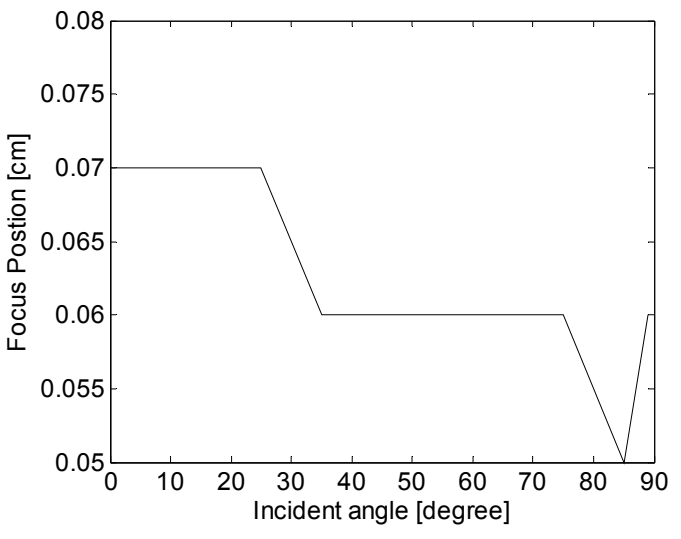

(c)

Figure 6. (a) Fluences at the effective optical focus and surface for the obliquely and cylindrical-symmetrically incident finite beam with $1 \mathrm{~mm}$ inner radius and $2.5 \mathrm{~mm}$ outer radius and incident angle ranging from 0 to 89 degree. (b) Ratio of fluences at the focus and surface as a function of incident angle. (c) Position of effective optical foci as a function of incident angle. 


\section{CONCLUSIONS}

In the present work, a modified MC convolution method for integration extension of MC simulation is developed for finite photon beam with random shape of translational and rotational invariance, which is proven consistent with the conventional convolution of MC simulation for normal incident finite beam. The method is applied to analyze the positions of effective optical foci and ratios of fluence at the focus and surface which are two key factors in the application of dark-field confocal PAM.

Taken together, the fluence distribution in dark-field confocal PAM has the following features: (1) The fluence profile has a saddle-like shape, the optical fluence is significantly reduced on the skin surface that is directly below the ultrasonic detector compared with bright-field illumination, thereby reducing the chances for reverberation; (2) Besides a little peak near zero inner radius, the ratio of fluences at the focus and surface increases linearly with the inner radius, suggesting the larger dark field leads to a higher imaging contrast.; (3) The position of effective optical foci deepens linearly with the increase of the inner radius, suggesting that to get a high quality image of deeper target, the dark-field with larger size is more beneficial. But the position of optical effective foci are far away from the foci of geometrical laser beam, so aligning the foci of geometrical laser beam and acoustic transducer doesn't guarantee that effective optical focus is accurately overlapping with the acoustic focus. An MC simulation with integration extension in advance maybe helpful to determine where the acoustic focus should be to maximize SNR in tissue imaging; (4) incident angle makes little difference to ratio of fluences at the focus and surface and a smaller incident angle may be slightly improve imaging quality. In terms of the highest fluence at the effective optical focus, an incident angle between 30-50 degrees is optimal; (5) the depth of fluence focus is insensitive to the incident angle.

\section{ACKNOWLEDGEMENTS}

This work was supported in part by The University of Wisconsin-Milwaukee start-up fund and by grant from The Lynde and Harry Bradley Foundation. We also thank Dr. Konstantin Maslov and Dr. Gang Yao for valuable discussions.

\section{REFERENCES}

[1] M. Xu, and L.-H. V. Wang, "Photoacoustic imaging in biomedicine," Rev. Sci. Instrum. 77, 041101-1-04110122(2006).

[2] L.-H. V. Wang, "Tutorial on photoacoustic microscopy and computed tomography," IEEE J. Sel. Topics Quantum Electron. 14(1), 171-179 (2008).

[3] K. Maslov, G. Stoica, and L. V. Wang, "In vivo dark-field reflection-mode photoacoustic microscopy," Opt. Lett. 30, 625-627 (2005).

[4] H. F. Zhang, K. Maslov, G. Stoica, and L. V. Wang, "Functional photoacoustic microscopy for high-resolution and noninvasive in vivo imaging," Nat. Biotechnol. 24, 848-851 (2006).

[5] H. F. Zhang, K. Maslov, M. Li, G. Stoica, and L. V. Wang, "In vivo volumetric imaging of subcutaneous microvasculature by photoacoustic microscopy," Optics Express 14, 9317-9323 (2006).

[6] H. F. Zhang, K. Maslov, M. Sivaramakrishnan, G. Stoica, and L. V. Wang, "Imaging of hemoglobin oxygen saturation variations in single vessels in vivo using photoacoustic microscopy," Appl. Phys. Lett. 90, 0539011-053901-3 (2007).

[7] H. F. Zhang, K. Maslov, and L. V. Wang, "In vivo imaging of subcutaneous structures using functional photoacoustic microscopy," Nat. Protocols 2, 797-804 (2007).

[8] L. V. Wang, and G. Liang, "Absorption distribution of an optical beam focused into a turbid medium," Appl. Opt. 38, 4951-4958 (1999).

[9] L.-H. Wang, S. L. Jacques, and L. Zheng, "MCML-Monte Carlo modeling of light transport in multi-layered tissues," Comput. Methods Programs Biomed. 47, 131-146 (1995).

[10] S. T. Flock, M. S. Patterson, B. C. Wilson, and D. R. Wyman, "Monte-Carlo modeling of light-propagation in highly scattering tissue. 1. Model prediction and comparison with diffusion theory," IEEE Trans. Biomed. Eng. 36, 1162-1168 (1989). 
[11] S. T. Flock, B. C. Wilson, and M. S. Patterson, "Monte-Carlo modeling of light-propagation in highly scattering tissue. 2. Comparison with measurements in phantoms," IEEE Trans. Biomed. Eng. 36, 1169-1173 (1989).

[12] S. A. Prahl, M. Keijzer, S. L. Jacques, and A. J. Welch, "A Monte Carlo model of light propagation in tissue," Proc. SPIE IS 5, 102-111 (1989).

[13] M. C. Skala, G. M. Palmer, K. M. Vrotsos, A. Gendron-Fitzpatrick and N. Ramanujam, "Comparison of a physical model and principal component analysis for the diagnosis of epithelial neoplasias in vivo using diffuse reflectance spectroscopy," Optics Express 15(12), 7863-7875 (2007).

[14] A. Wang, V. Nammalvar and R. Drezek, "Targeting spectral signatures of progressively dysplastic stratified epithelia using angularly variable fiber geometry in reflectance Monte Carlo simulations," J. Biomed. Opt. 12(4), 044012-1-044012-14 (2007).

[15] D. Arifler, C. MacAulay, M. Follen and R. Richards-Kortum, "Spatially resolved reflectance spectroscopy for diagnosis of cervical precancer: Monte Carlo modeling and comparison to clinical measurements," J. Biomed. Opt. 11(6), 064027-1-064027-16 (2006).

[16] R. K. Wang, "Signal degradation by multiple scattering in optical coherence tomography of dense tissue: A Monte Carlo study," Phys. Med. Biol. 47, 2281-2299 (2002).

[17] Z. Song, K. Dong, X. H. Hu, and J. Q. Lu, "Monte Carlo simulation of converging laser beams propagating in biological tissue," Appl. Opt. 37, 2944-2949 (1999).

[18] J. Q. Lu, X. H. Hu, and K. Dong, "Modeling of the rough-interface effect on a converging light beam," Appl. Opt. 39, 5890-5897 (2000).

[19] L. Wang, S. L. Jacques, and L. Zheng, "CONV-convolution for responses to a finite diameter photon beam incident on multi-layered tissues," Comput. Methods Programs Biomed. 54, 141-150 (1997).

[20] Q. Liu, and N. Ramanujam, "Scaling method for fast Monte Carlo simulation of diffuse reflectance spectra from multilayered turbid media," J. Opt. Soc. Am. A 24(4), 1011-1025 (2007).

[21] Laser Inst. Amer., "American National Standard for Safe Use of Lasers," ANSI Standard Z136.1-2000, NY, (2000). 